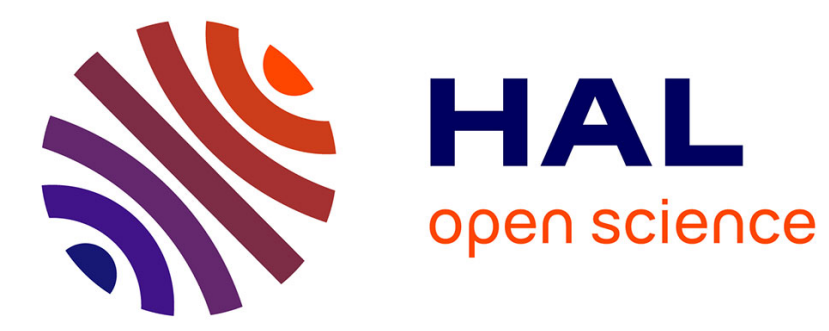

\title{
Explaining with Models: The Role of Idealizations
}

Julie Jebeile, Ashley Graham Kennedy

\section{To cite this version:}

Julie Jebeile, Ashley Graham Kennedy. Explaining with Models: The Role of Idealizations. International Studies in the Philosophy of Science, 2015, 29 (4), pp.383-392. 10.1080/02698595.2015.1195143. hal-02105520

\section{HAL Id: hal-02105520 \\ https://hal.science/hal-02105520}

Submitted on 23 Apr 2019

HAL is a multi-disciplinary open access archive for the deposit and dissemination of scientific research documents, whether they are published or not. The documents may come from teaching and research institutions in France or abroad, or from public or private research centers.
L'archive ouverte pluridisciplinaire HAL, est destinée au dépôt et à la diffusion de documents scientifiques de niveau recherche, publiés ou non, émanant des établissements d'enseignement et de recherche français ou étrangers, des laboratoires publics ou privés. 


\title{
Explaining with models: the role of idealizations
}

Julie JEBEILE (IHPST, Paris) and Ashley KENNEDY (Florida Atlantic University) ${ }^{1}$

\begin{abstract}
Because they contain idealizations, scientific models are often considered to be misrepresentations of their target systems. An important question is therefore how models can explain the behaviors of these systems. Most of the answers to this question are representationalist in nature. Proponents of this view are generally committed to the claim that models are explanatory if they represent their target systems to some degree of accuracy; in other words, they try to determine the conditions under which idealizations can be made without jeopardizing the representational function of models. In this paper we first outline several forms of this representationalist view. We then argue that this view, in each of these forms, omits an important role of idealizations: that of facilitating the identification of the explanatory components within a model. Via examination of a case study from contemporary astrophysics, we show that one way in which idealizations can do this is by creating a comparison case which serves to highlight the relevant features of the target system.
\end{abstract}

\section{Introduction}

All scientific models contain idealizations, which are deliberate distortions or omissions of specific properties of the target system. Often, these idealizations are required for mathematical tractability. Because of the idealizations, scientific models are often considered to be "misrepresentations" of their targets. On the surface, it might seem that a misrepresentation would not provide a good explanation. However, most scientists, and indeed most philosophers of science, take idealized models to be explanatory. Exactly how these models explain, however, is controversial.

\footnotetext{
${ }^{1}$ Both authors have contributed equally to this work. Julie Jebeile is at the Institute for the History and Philosophy of Science and Technology (IHPST). Correspondence to: IHPST - UMR 8590, 13 rue du Four, 75006 Paris, France. E-mail: julie.jebeile@gmail.com. Ashley Kennedy is at Florida Atlantic University. Correspondence: 5353 Parkside Dr., Jupiter, FL 33458. E-mail: kennedya@fau.edu.
} 
For the most part, attempts to understand model explanation have centered on an analysis of scientific models as representations. One such attempt is the de-idealization thesis which is most famously represented in McMullin (1985), but is also present in Laymon (1987) and Nowak (1992). Other representationalist attempts have been more recently provided and/or discussed by Strevens (2008), Bokulich (2009, 2011), Mäki (2009), Morrison (2009), and Weisberg $(2007)^{2}$. Even though what exactly representation amounts to is the subject of ongoing debate (see van Fraassen 2008), these authors seem to be generally committed to the claim that models are explanatory if they represent their target systems to some degree of accuracy. No doubt this presumption stems from Hempel's condition of truth (which he refers to as the "empirical condition of adequacy") in the deductive-nomological account of scientific explanation (1965), which requires that "the sentences constituting the explanans must be true." Thus these representationalist authors generally try to determine the conditions under which idealizations can be employed without jeopardizing the representational function of the model that contains them.

Our aim in this paper is not to argue against the representationalist account of model explanation but rather to show one way in which it is incomplete and in need of revision. Our view is that a strictly representationalist account of model explanation does not do justice to the role of idealization in model explanation. In order to show this, we will first outline the prevailing representationalist accounts of model explanation, then we will argue that these accounts omit an important role of idealizations: that of facilitating the identification of the components within a model that do real explanatory work. Further, we will argue that explanation should be understood as an activity, rather than a product. In order to see both of these points, it is necessary to consider not only the dyadic relationship between models and target systems, but also the relationship between models and users. Models are not only representations, they are also epistemic tools which are designed by and for scientists to make inferences, predictions and explanations. Authors such as Giere (2010), Vorms (2011) and Suárez (2010) have recently developed accounts of scientific representation, which describe a role for the scientist or model "user" in representation. Under this kind of framework, as we will show, idealizations cannot be understood as merely having a negative role in possibly jeopardizing the representational function of models. Rather, on the contrary, idealizations should be seen as having an active role in making possible the identification of explanatory

\footnotetext{
${ }^{2}$ Non-representationalist accounts of model explanation have also been developed (Elgin 2007, 2009; Kennedy 2012; Knuutila and Merz 2009, Knuuttila 2011).
} 
components in models; a role that current representationalist accounts of model explanation do not account for. Via examination of a case study from contemporary astrophysics, we will show one way in which idealizations can play such an active role in model explanation: by creating a comparison case which highlights the explanatorily relevant features of the target system. This example further shows that explanation is a process or an activity, rather than simply a product.

\section{Representationalist accounts of model explanation}

\subsection{The de-idealization thesis}

According to one representationalist account - the "de-idealization thesis" - a model can be considered explanatory only if, were it to undergo a process of de-idealization, it would be a more accurate representation of (and thereby would provide a better explanation of) the target system in question (McMullin 1985; Laymon 1995; Jones 2005; Nowak 1992). On this view, a process of "de-idealization" consists in adding features of the target (that were originally left out) back in to the models and/or correcting mathematical approximations that originally appear in the models. According to this thesis, if a model undergoes such a process of deidealization and becomes more accurate or "more specific" (McMullin 1985), it means that the model in its idealized form was already a partially or approximately true representation of the target, and can therefore be considered explanatory.

The aim of the corrective, or de-idealization process, (McMullin, 1985) is to create more accurate models by adding back in features of the target system which were originally removed, or by replacing initially highly simplified terms by more approximate ones. Idealizations which are amenable to being corrected in this way are qualified as "Galilean". Examples of Galilean idealizations include the frictionless plane, the simple pendulum and inviscid fluid flow. According to this view, only a model that can be de-idealized, via a correction of its Galilean idealizations, is likely to be approximately true and therefore has the potential to explain the structure of the target system in question. Hence, only such a model can serve "as the basis for a continuing research program" (McMullin, 1985, p. 261). The deidealization process, according to McMullin, justifies the explanatory value of a model, because if a model can be de-idealized, that means that it captures what he calls the "real 
structure" of the target system that it represents ${ }^{3}$. Therefore, on this view, de-idealization is seen as a way of enhancing or improving a model, and thus is considered to be a central part of the development of the research program. According to the de-idealization thesis, then, the aim of the modeling process is to develop models that are increasingly realistic so that we can arrive at increasingly better explanations of the systems that we model.

We think that there is something right about this view of the modeling process. In some cases, creating a more realistic model via de-idealization does in fact improve the model explanation. Take, for example, Bohr's original model of the atom (cf. McMullin 1985), which represented an electron in a circular orbit around a stable proton. This famous model, although very simplified, yielded "some startling good results for the basic H-spectrum." (McMullin 1985 p. 260) The model was made simple and tractable by incorporating three main idealizing assumptions. First, it assumed that the orbit of the electron was circular. Next, it assumed that the nucleus of the atom was at rest (and thus of infinite mass) and finally, it ignored the relativistic effects due to the rapid motion of the electron. These idealizing assumptions were made for pragmatic reasons, even though they were known to be false. Later, when this model was improved by introducing corrective terms for each of its original idealizations, the de-idealized versions of the original model were considered to be more explanatory than their predecessor.

This particular example well supports the claim of the de-idealization thesis - that deidealizing a model can improve the model explanation ${ }^{4}$. However, as we will show, a deidealized version of a model is not always in itself explanatory beneficial. Before we make

\footnotetext{
${ }^{3}$ Note that McMullin $(1978,1985)$ describes a kind of explanation that is structural. He writes that "When the properties or behavior of a complex entity are explained by alluding to the structure of that entity, the resultant explanation may be called a structural one." (1978, p.139) McMullin takes this kind of explanation to be causal, since the structure that is identified is generally the cause of the feature that is being explained.

${ }^{4}$ That said, Morrison $(2005,2009)$ showed that there is a theoretical inconsistency in the de-idealization thesis. According to the de-idealization thesis, if a model can be de-idealized, then it is a good approximate representation: it is approximately true. And the more we de-idealize the model, the better the model explanation will be. However, this is only possible if there is a stable "structure," or set of approximately true assumptions, that remains constant throughout the de-idealization process. As Morrison points out, the problem is that such a structure does not always exist. In some cases, a model that results from a de-idealization process will contradict the model from which it was derived, which seems to show that there is no constant underlying structure that survives the de-idealization process. While we agree with Morrison that there does seem to be a theoretical inconsistency in the de-idealization thesis, we think that the following question is still worth addressing: in practice, even if successive de-idealized models conflict with each other, is de-idealization always explanatorily beneficial? And we argue, in the remaining of the paper, that a de-idealized version of a model is not always in itself explanatorily beneficial.
} 
this argument, we will first examine some more recent representationalist accounts of model explanation.

\subsection{More recent representationalist accounts}

Recently, Elgin and Sober have claimed that: "The idealizations in a causal model are harmless if correcting them wouldn't make much difference in the predicted value of the effect variable". (2002 p. 447). In other words, they argue that a model is explanatory insofar as the idealizations it contains do not make much difference between the explanation provided by the idealized model and the one provided by the corrected model.

Strevens (2008 p. 316) has similarly argued that all idealizations "work in the same way: an idealization does not assert, as it appears to, that some non-actual factor is relevant to the explanandum, rather, it asserts that some actual factor is irrelevant." Thus, on his view, the role of idealization in model explanation is to highlight those factors that are not causally relevant to the target being explained. While we agree that sometimes idealizations within a model do play this role, we will argue that, in other cases, the idealizations within scientific models play a more active role.

According to another recent account of model explanation, (Bokulich's 2009, 2011), models are explanatory not in spite of their idealizations, but in virtue of them. Bokulich argues that in order for a model to count as explanatory its components must correctly capture the structure of counterfactual dependences in the target system. This means that the counterfactual structure of the model must be in an isomorphic relation with the target system. Bokulich bases her account on Woodward's interventionist account of scientific explanation (2003). She also specifies that the isomorphic relation between the model and the target system must be established in accordance with the relevance of the system's features. In other words, the components of the model must refer to the relevant features of the system. The relevance of these features is determined by the type of questions that the model aims to answer. A model that relevantly captures the genuine counterfactual structure of the system therefore allows one to answer a broad class of "what-if-things-had-been-different" questions.

All of these views of model explanation are alike in that they posit that scientific models explain, at least in part, because they are good representations of their target systems. However, as we will argue, representation alone is not enough for explanation. 


\section{Model explanation as an activity}

Our view is that representation is not sufficient for explanatory sucess. Instead, in order for a model to be successfully explanatory, the scientists who employ the model must be able to draw explanations from it. And for this purpose, scientists, in some cases, require idealized representations, in addition to the most accurate representation, in order to explain their targets. However, the representationalist assumption falls short in accounting for this kind of scientific activity since it only allows for accurate representations to be explanatory, and ignores all the other means - such as the use of more idealized representations - used by scientists to answer their explanatory questions. Our goal in this paper is to revise the representationalist view by taking into account that explanation is an activity. In this regard our account of explanation is closely related to Suárez's (2015) account of representation. He writes that in scientific modeling, "It is the activity itself that is constitutive of representation, and there is no relation that may stand in its place." Similarly, in our view, explanation cannot be reduced in this way either.

On our view, the success of the activity of explaining depends upon several factors. Here we will discuss two. First, the activity of explaining with a model requires that the model not only represent its target, but also that it allow those who use it to extract relevant pieces of information about the target system from the model. In most cases this requires that the model be an idealized representation of its target in appropriate ways. A completely realistic model, even if it were possible to create, would not allow for the extraction of information leading to explanation. Second, the success of a model explanation also depends upon the skills of an experienced scientist to draw explanations from the model. This means that no model is, on our view, explanatory per se, but that certain models can become explanatory when in the hands of qualified users. In other words, model explanation is a process that is user dependent.

As we have noted, there is an extensive literature on models and scientific representation. Much of this literature seems to either implicitly or explicitly assume that models explain when they are accurate (or at least adequate) representations of their target systems. This view leaves little room for the role of idealization in model explanation, other than the "causal isolationist" view according to which idealization within models is a means to isolating the causal mechanisms that are at work in the target system that is being modeled. On this view, scientists include idealizations as a way of sealing off factors that are considered irrelevant to 
what is being explained. Weisberg (2007) refers to these models as "minimalist models," which are models that contain "only those factors that make a difference to the occurrence and essential character of the phenomenon in question." (2007, p. 4). In some cases, scientists do use idealizations for this purpose, as a way of pointing out that certain factors are not relevant to what is being explained. For example, friction and air resistance are often given a zero value in models, not just for tractability reasons, but because friction and air resistance, in many cases, are not relevant to what is being explained.

Another role for model idealization that has been described in the literature is that of simplification for the purposes of facilitating understanding. We agree that idealizations do sometimes play this kind of role, by simplifying very complex models in order to make them understandable to users ${ }^{5}$. But while we acknowledge that idealizations in scientific models can be used to simplify these models, what we wish to highlight here is that, at least in some cases, idealization can play another role in the explanatory process. In the example that follows, the model explanation is derived, not just from the representationally accurate parts of the model, but also via a comparison of the idealized model with more realistic versions. Thus the idealizations in this case plays an important role in the process of explanation that goes beyond mere simplification.

Our second point concerning the process of explanation is that it is user-dependent. We have said that explanation can be conceived of as an activity. This activity depends, for its success, upon the skills of a competent model user to draw inferences from the model in question. Because of this, model explanation cannot, in our view, be understood independently from the user of the model. In other words, explanation requires a competent model user.

To see our view more clearly, consider the following example.

\footnotetext{
${ }^{5}$ While many philosophers of science focus on the epistemic problem raised by simplifying idealizations in models, some have focused on their role as cognitive aids (Dilworth (ed.) 1992; Elgin 2007, 2009; Forster 2001; Hartmann 1998; Morrison 2009; Teller 2001). In particular, Suárez (2009, 2010) contends that fictional assumptions play an inferential role in scientific modeling. On his view the primary function of a scientific model is not (only) to faithfully represent the target system but also to provide "inferential shortcuts" from which we can access the properties of the target.
} 


\section{Case study: cosmological hydrodynamical simulations}

The standard model of cosmology posits that the mass-energy density of the Universe is dominated by dark matter and dark energy of unknown forms. Because dark matter is not directly observable, testing the predictions of the standard cosmological model requires observing matter in the form of stars, diffuse gas, and accreting black holes. These components of visible matter are organized in sheets, filaments, and voids, inside of which galaxies are embedded. Because galaxies are the basic units of cosmological structure, simulating and observing galaxies is a way in which to test the predictions of the standard cosmological model. Thus scientists create simulated galaxies and then compare the predictions of these simulations to observational data of actual galaxies. They then evaluate the successes and failures of these simulations in order to enhance understanding of the galaxy formation process and ultimately of cosmological structure. As with accretion disk modeling, the comparison of idealized with more realistic models is an integral part of the explanatory process in these cases.

As we have noted, galaxy simulations are important for understanding large scale cosmology. For practical reasons, these galactic simulations are often idealized in several ways. In particular, a recent study (Puchwein et. al. 2015) compared galaxy simulations that assume that the intergalactic medium (IGM) is in photoionization equilibrium ${ }^{6}$ (equilibrium simulations) with more realistic simulations that drop this simplifying assumption (nonequilibrium simulations). The study then compared the temperature predictions of both of these simulations with observed temperatures in the Lyman-alpha forest ${ }^{7}$. The authors of this study reported that:

the non-equilibrium treatment results in a much larger temperature increase during the almost simultaneous Hi and He i reionization between red-shifts $\sim 15$ and $\sim 12$, as well as during He ii reionization between redshifts $\sim 5$ and $\sim 3.5$. In the equilibrium treatment, an increase in the photoionization rates results in an unrealistic instantaneous increase of the ionized fractions as they are directly set to the new equilibrium value. [...] The significant differences in the temperature re-emphasizes

\footnotetext{
${ }^{6}$ Photoionization refers to the state of complete (or near complete) ionization of hydrogen.

${ }^{7}$ The Lyman alpha forest is an absorption phenomenon seen in the spectra of high red-shift galaxies.
} 
the potential importance of accounting for out-of-equilibrium ionized fractions in cosmological hydrodynamical simulations. (Puchwein et al. 2015, p. 4086)

In other words,

A comparison of our equilibrium and non-equilibrium simulations corroborates previous findings that non-equilibrium effects are indeed significant. [...] They, thus, ideally need to be taken into account in cosmological hydrodynamical simulations. (Puchwein et al. 2015, p. 4091)

Thus the scientists in this case made use of both idealized and de-idealized simulations simultaneously in order to interpret and explain their modeled targets. In this case, the comparison of the two simulations with each other, as well as with observational data, allowed the scientists to confirm that equilibrium status is important in galactic temperature change. This example shows that, de-idealized models, in themselves, are often not enough for explanation of a target system. Instead, the activity of comparison, which depends upon the use of idealization, in some cases plays an important explanatory role. In the example, the scientists were able to confirm that temperature does in fact depend upon the ionization status of the galactic gas by comparing the idealized and the de-idealized galactic simulations.

\section{The activity of explaining with models}

The comparison between the idealized model and its de-idealized counterpart that we have described in the example might be seen by some as a species of what is known in the literature as contrastive explanation. In particular, Lipton $(1991)^{8}$ talks about contrastive cases in his account of "Inference to the Best Explanation." The general idea is that claims of the form " $p$ explains q" are elliptical for " $p$ (rather than $p^{*}$ ) explains $q$ (rather than $\left.q^{*}\right)$ ". Lipton uses contrastive cases as "fact" and "foil" where "X1 explains Y1 rather than X2 explains Y2." In our example, one might think that the idealized model acts as a kind of foil for the deidealized one, that it is the de-idealized model that is doing the explaining by transmitting the information that embodies the explanatory mechanism, and that the idealized model provides a kind of epistemic support system that illustrates the power of the de-idealized model. Nevertheless our account of explanation by comparison differs from Lipton's account in that

\footnotetext{
${ }^{8}$ Contrastive explanations were first systematically developed by Alan Garfinkel (1981) and Bas van Fraassen (1980).
} 
the comparison that we describe should be considered as an explanatory activity - and one that serves the users to highlight what is relevant to the phenomena being explained. In addition, the activity of comparing idealized with de-idealized models that we have identified calls into question the model-oriented version of the Duhem-Quine thesis (Lenhard and Winsberg 2010, Winsberg 2010). According to this version of the thesis the different theoretical assumptions of a model cannot be tested separately. However, with the sort of comparative work in scientific modeling that we have here described, model assumptions can indeed be isolated and studied separately ${ }^{9}$. This allows scientists to study their respective effects (relative to the results of the more idealized versions of the model), and to say whether the assumption under consideration is a genuinely explanatory element of the model. In the example, the effect of an equilibrium assumption on galactic simulation models was only fully understood by comparing a model that included this assumption with a model that did not. Thus idealization was required in the process of successful model explanation.

This example ${ }^{10}$ thus allows us to go beyond strictly representationalist accounts of model explanation in the following way. It shows that at least in some cases, de-idealization alone does not improve itself the explanatory power of a model. In cases such as this, idealized models are required in the activity of explanation.

\section{Conclusion}

In this paper, we argued that in some cases, the activity of comparison between an idealized and a de-idealized model is pivotal in generating a successful model explanation. Thus we argued that a strictly representationalist view of model explanation, while certainly plausible in many instances, does not allow for a complete understanding of the various roles that idealization can play in model explanation. Instead our claim is that there is an additional role, beyond that of simplification or isolation, that idealization can play in model explanation: that of facilitating the identification of the explanatory components within a model. While computer-assisted science increasingly allows for scientific models to be de-idealized, we

\footnotetext{
${ }^{9}$ The comparative work that we have described in our examples is not limited to de-idealization. Such comparative work might be also done by scientists when they replace the laws in their initial model by ones that are considered to be more fundamental, in an effort to improve their models.

${ }^{10}$ We use an example from physics, however, explanation by comparison occurs in other disciplines as well, such as economics (see Lehtinen and Kuorikoski 2007; Lehtinen 2013 for examples).
} 
think that idealizations will always play an important role in model explanation. This idea is shared by other philosophers such as Hartmann (1998) who writes: that, "even when we can solve whatever equation we want on a computer, physicists will certainly not stop investigating idealized models. This is because of the cognitive role of idealizations. Idealized models give us a partial understanding of the relevant mechanisms for the processes in the system under study.” (p. 118)

\section{Acknowledgments}

Previous versions of this work have been presented at the Society for Philosophy of Science in Practice conference held in Toronto, June 2013, and at the Biennial Meeting of the Philosophy of Science Association held in Chicago, November 2014. We thank the audience for their comments. In particular, we would like to thank Anouk Barberousse, Aki Lehtinen, Mauricio Suárez and the two anonymous reviewers for their precious comments.

\section{References}

Bokulich, A. (2009). Explanatory Fictions. In M. Suárez (Ed.) Fictions in Science: Philosophical Essays on Modeling and Idealization, (pp. 91-109).Routledge, London.

Bokulich, A. (2011). How Scientific Models Can Explain. Synthese, 180(1):33-45.

Dilworth, C. (Ed.) (1992). Idealization IV: Intelligibility in Science. Poznan Studies in the Philosophy of the Sciences and Humanities. Amsterdam/New York, NY: Rodopi.

Elgin, C. (2007). Understanding and the facts. Philosophical Studies, 132: 33-42.

Elgin, C. (2009). Telling Instances. In R. Frigg (Ed.) Beyond Mimesis and Convention. Dordrecht: Springer. pp. 1-18

Elgin, M., \& Sober, E. (2002). Cartwright on Explanation and Idealization. Erkenntnis, 57(3):441-450.

Forster, M. R. (2001). The New Science of Simplicity. In A. Zellner, H. Keuzenkamp, \& M. McAleer (Eds.) Simplicity, Inference and Modelling, (pp. 83-117). Cambridge: Cambridge University Press. 
Garfinkel, A. (1981). Forms of Explanation: Rethinking the Questions in Social Theory. New Haven: Yale University Press.

Giere, Ronald. (2010) "An Agent-Based Conception of Models and Scientific Representation." Synthese: 172:269-281.

Hartmann, S. (1998). Idealization in Quantum Field Theory. In N. Shanks (Ed.) Idealization in Contemporary Physics, (pp. 99-122). Rodopi: Amsterdam.

Hempel, C. G. (1965). Aspects of Scientific Explanation. In Aspects of Scientific Explanation, and Other Essays in the Philosophy of Science, (pp. 331-496). Free Press.

Jones, M. R. and Cartwright N. (eds.) (2005) Idealization XII: Correcting the Model. Idealization and Abstraction in the Sciences. Amsterdam/New York, NY: Rodopi.

Jones, M. R. (2005) "Idealization and Abstraction: A Framework," in Jones, M.R. and Cartwright, N. (2005), pp.173-217

Kennedy, A. (2012) A Non Representationalist View of Model Explanation. Studies in History and Philosophy of Science. Volume 43, Issue 2, pp. 233-420.

Knuuttila, T., \& Merz, M. (2009). Understanding by Modeling: An Objectual Approach. In H. de Regt, S. Leonelli, \& K. Eigner (Eds.) Scientific Understanding: Philosophical Perspectives, chap. 8, (pp. 146-168). University of Pittsburgh Press.

Knuuttila, T. (2011) "Modelling and Representing: An Artefactual Approach to Model-Based Representation.” Studies in History and Philosophy of Science.

Laymon, R. (1987). Using Scott Domains to Explicate the Notions of Approximate and Idealized Data. Philosophy of Science, 54:194-221.

Laymon, R. (1995). Experimentation and the Legitimacy of Idealization. Philosophical Studies, 77(2-3):353-375.

Lehtinen, A. (2013). Three Kinds of 'as-if' Claims, Journal of Economic Methodology vol. 20, no. 2, pp. 184-205.

Lehtinen, A. \& Kuorikoski, J. (2007). Unrealistic Assumptions in Rational Choice Theory, Philosophy of the Social Sciences, vol. 37, no. 2, pp. 115-138.

Lenhard, J., \& Winsberg, E. (2010). Holism, Entrenchment, and the Future of Climate Model Pluralism. Studies in History and Philosophy of Science Part B, 41(3):253-262. 
Lipton, P. (1991) Contrastive Explanation and Causal Triangulation. Philosophy of Science, Vol. 58, No. 4, pp. 687-697.

Mäki, U. (2009) "Missing the world: models as isolations and credible surrogate systems." Erkenntnis, 70:29-43.

McMullin, E. (1978). Structural Explanation. American Philosophical Quarterly, 15(2):139147.

McMullin, E. (1985) “Galilean idealization.” Studies In History And Philosophy of Science, 16 (3):247-273.

Morrison, M. (2005). Approximating the real: the role of idealizations in physical theory. In N. Cartwright, \& M. R. Jones (Eds.) Idealization XII: Correcting the model : idealization and abstraction in the sciences, (pp. 145-171). Cambridge, UK: Cambridge University Press.

Morrison, M. (2009). Understanding in Physics and Biology: From the Abstract to the Concrete. In H. de Regt, S. Leonelli, \& K. Eigner (Eds.) Scientific Understanding : Philosophical Perspectives, chap. 7, (pp. 123-145). University of Pittsburgh Press.

Nowak, L. (1992). “The idealizational approach to science: A survey.” In Brzezinski, J. and Nowak, L., Idealization III: Approximation and Truth, volume 25 of Poznán Studies in the Philosophy of the Sciences and the Humanities, (pp. 9-63). Rodopi, Amsterdam and Atlanta, GA.

Puchwein, E., J. S. Bolton, M. G. Haehnelt, P. Madau, G. D. Becker, and F. Haardt. (2015). "The Photoheating of the Intergalactic Medium in Synthesis Models of the UV Background." Monthly Notices of the Royal Astronomical Society 450: 4081-4097.

Strevens, M. (2008). Depth: An Account of Scientific Explanation, Harvard UP. Chapter 8

Suárez, M. (2009). Scientific Fictions as Rules of Inference. In M. Suárez (Ed.) Fictions in Science: Philosophical Essays on Modeling and Idealization, (pp. 158-178). London: Routledge.

Suárez, M. (2010). Fictions, Inference and Realism. In J. Woods (Ed.) Fictions and Models: New Essays, (pp. 225-245). Munich: Philosophia Verlag.

Suárez, M. (2015). Deflationary representation, inference, and practice. Studies in History and Philosophy of Science. 49: 36-47.

Teller, P. (2001). Twilight of the Perfect Model Model. Erkenntnis, 55:393-415. 
van Fraassen, B. (1980). The Scientific Image. Oxford: Clarendon Press.

van Fraassen, B. C. (2008). Scientific Representation: Paradoxes of Perspective. Oxford University Press.

Vorms, M. (2011). Representing with Imaginary Models: Formats Matter. Studies in History and Philosophy of Science, 42(2):287-295.

Weisberg, M. (2007). Three Kinds of Idealization. Journal of Philosophy, 104(12):639-659.

Winsberg, E. (2010). Science in the Age of Computer Simulation. The University of Chicago Press.

Woodward, J. (2003). Making Things Happen: A Theory of Causal Explanation. Oxford University Press. 